PSYCHOMETRIKA-VOL. 6, NO. 5

OCTOBER. 1941

\title{
A FACTOR ANALYSIS OF VERBAL ABILITIES*
}

\author{
JOHN B. CARROLL
}

MOUNT HOLYOKE COLLEGE

\begin{abstract}
A multiple-factor analysis was made of a battery of 42 tests of verbal abilities administered to 119 college adults. Where necessary, the distributions of test scores were normalized before the inter-test correlations were computed. Thurstone's $M$ (Memory or Rote Learning) factor has been confirmed, but his $V$ (Verbal Relations) factor seems to have been split into two or possibly three factors, $C, J$, and G; and his $W$ (Word Fluency) factor has been split into two factors, $A$ and $E$. The $C$ factor seems to represent the richness of the individual's stock of linguistic responses, and the $J$ factor seems to involve the ability to handle semantic relationships. No satisfactory interpretation can as yet be made of the $G$ factor. The $A$ factor seems to correspond to the speed of association for common words where there is a high degree of restriction as to appropriate responses. The $E$ factor is described as an associational facility with verbal material where the only restriction is that the responses must be syntactically coherent. The new factors are: $F$, facility and fluency in oral speech; $H$, facility in attaching appropriate names or symbols to stimuli; and D, speed of articulatory movements.
\end{abstract}

The purpose of the present investigation has been to explore the domain of speech and language behavior by means of Thurstone's multiplefactor analysis (10) (13). Although the present study has taken as its starting point certain results of Thurstone (11) which bear on verbal abilities, an attempt has been made to examine as broad an area in this domain as possible. The study has been exploratory in character, and the writer has been more interested in obtaining an approximate delineation of the field than in answering the detailed problems which inevitably present themselves. No investigator has attempted a comprehensive examination of the field of speech and language abilities, although the problem of the linguistic factors in what is known as "intelligence" has received considerable attention.

A major problem has been the further definition of the $V$ (Verbal Relations) and $W$ (Word Fleuncy) factors isolated by Thurstone (11) (12) (14). Although the $V$ factor has often been specified as one of the clearest factors in the previous studies, tests having high saturations on this factor have been relatively so diversified that it has not been possible to make such a simple hypothesis regarding the nature of the factor as has been possible in the case of certain other factors. Regarding the verbal factor, Thurstone has committed him-

* This paper is a condensation of the writer's doctoral dissertation, "A Factor Analysis of Verbal Abilities," on file at the library of the University of Minnesota. 
self to stating only that "the factor is evidently characterized primarily by its reference to ideas and the meanings of words" and that "it is quite likely, as far as one can judge from the present data, that the factor $V$ will be identified largely in terms of the verbal manipulation of ideas as they occur in sustained verbal discourse" (11, pp. 84-85). The writer believes that it would be desirable to describe the verbal factor in terms of some kind of psychological process rather than merely in terms of the type of material with which the factor is associated. Furthermore, it is not certain that the interpretations of the verbal factor which have been advanced thus far are of sufficient generality. Thurstone has suggested that in order to resolve this difficulty comparisons should be made between tests which involve the manipulation of ideas in verbal and in essentially nonverbal form (11, p. 85). Nevertheless, the writer has not been able to conceive tests which clearly involve ideas in nonverbal form, with the possible exception of a syllogism test utilizing Euler's circles. It might be possible to construct a verbal analogies test or even a vocabulary test in pictorial form, but even if this were done there would remain the possibility that the solution of problems cast in nonverbal form would involve implicit verbalization on the part of the subject. On theoretical grounds, it would seem that the essentially verbal character of "ideas" would not permit their appearance in any other than verbal tests. The present study has not attempted to make a direct solution of the question of the verbal or nonverbal character of the $V$ factor; it was thought, however, that in view of the extent and diversity of the test material, the results might suggest a proper mode of attack on this problem in future work.

Throughout the previous studies of the primary mental abilities, the interpretation of the factor $W$ has remained somewhat doubtful. In the 57test battery of Thurstone (11), the highest $W$ saturations were found for tests in which the subject deals with single and isolated words, usually without regard to the meanings of these words. In later studies, the single-word feature of the $W$ tests was again noticed, but there was a suspicion that this was merely a coincidence. The tests seemed to fall into two general types: (1) tests which involve words in which the letters are disarranged, and (2) tests which require the subject to think of appropriate words in a given situation - for example, any words having to do with food, or any words having the suffix -able. Wherever a factor seems to embrace two fairly distinct types of psychological functions there is the possibility that the test batteries have lacked pure tests of these respective functions and that consequently the dimensionality of the factor system has been too low. In such a case a new factor study should seek to split 
up the doubtful factor by attempting to find pure tests of each function. The present study included as many types of $W$ tests as possible, but as in the case of the $V$ factor it did not attempt to test any simple hypotheses regarding this factor, for the reason that such hypotheses did not seem available. The only hypothesis which was considered in assembling the battery was to the effect that the $W$ factor is an associational facility with familiar and common words.

The general plan of the study has embraced a large number of subsidiary problems. Many of the detailed questions asked in this investigation will be more conveniently mentioned in connection with the test battery, but it will be useful to discuss here several of the more general problems.

One of these problems was to determine the place, in the domain of verbal abilities, of the oral speech abilities involved in everyday communication. It was sought to discover whether what may be called "general speech fluency" or more popularly "gift of gab" is an operational unity unrelated to intellectual abilities as represented by Thur-stone's $V$ and $W$ factors. Thurstone has suggested $(11, \mathrm{p}$. 85) that the $W$ factor is associated with some sort of verbal fluency, though he has not included tests of speech ability in his experimental batteries of written group tests, since such tests are of necessity administered individually. The present study has also attempted to discover in what way the quality and the quantity of speech behavior are differentiated and to what extent such variables as confidence in speaking and oral motor skill are important in this area of behavior.

The problems of ability in written composition and general facility in writing are somewhat similar to those of speaking ability. We may ask whether there is an operational unity, "facility and readiness in writing," which is independent of previously discovered factors. A negative answer to this question is suggested by the fact that the quality of written composition as rated by English teachers was found by Thurstone to have an appreciable loading (.357) on the $V$ factor (11). A hypothesis which the present study was expected to test was that the number of words written in a theme is to some extent a function of simple speed of handwriting. It was also thought that speaking and writing ability may have in common something which may be described as the ability to organize the elements of complex stimulus situations in coherent verbal form.

Finally, it has been the hope of the writer that the identification and interpretation of the primary abilities involved in speech and language behavior will eventually lead to a better understanding of the mental processes and psychological laws underlying verbal behavior in human beings. 
It was necessary to include in the experimental battery a number of tests which would define certain factors which had been previously established by the studies of Thurstone and others and which were considered relevant to our problems. It would have been desirable to have included tests of all the previously identified primary mental abilities... and if it had been feasible the writer might have used the machine scored Testa for Primary Mental Abilities, issued by the American Council on Education. The latter tests, however, require a total testing time of some five hours. Since the writer's testing time was limited, only three primary factors, $V . W$, and $M$, were selected for inclusion in the battery.

The factor $V$ (Verbal Relations) was represented by Thurstone's tests Inventive Opposites (G-26) *, Verbal Analogies (G-20), and Grammar (G19). New tests which were expected to involve the $V$ factor were Morpheme Recognition (G-30, G-31), Vocabulary (G-36), Distorted English (G-37), and Nonsense Numbers (I-56). These are described below. Thurstone's $W$ (Word Fluency) factor was represented by Disarranged Words (G-25) and Anagrams (G-23), both copied, with minor modifications, from Thurstone's 57-test battery (11). In addition, Disarranged Words II (G-13), Suffixes (G22), Rhyming (G-24), and Disarranged Morphemes (G-40), all constructed by the writer, were included to test certain hypotheses concerning the $W$ factor. The $M$ (Memory or Rote Learning) factor was represented by Thurstone's Word-Number test (G-39) and by a Paired Associates test (G34, G-35) constructed by the writer.

The tests finally assembled in the present battery, whether taken from previous sources or constructed by the writer, are listed and described below.

Disarranged Words II (G-13) was prepared by the writer as a test of the $W$ factor. It is similar to Thurstone's Disarranged Words test (included in the present battery as G-25) except that no clue is given as to the meanings of the words whose letters are disarranged.

Free Writing (G-14, G-15. G-16) is similar to Thurstone's Theme Writing test (11, test no. 52), but whereas Thurstone asked his subjects to describe a friend or acquaintance, the writer set the task of writing a theme on the international situation. Three scores are derived from the themes, identified as follows: G-14 is a composite rating, made by a number of competent judges, of the excellence of the theme apart from the amount of information exhibited by the subject

* Each test (or score on a test) is identified by a code number throughout the present paper. The letter $G$ is prefixed to the code numbers of group tests, the letter I to those of individual tests. 
and the merits of his opinions. G-15 is the raw number of running words written in the theme. G-16 is the number of different words in the first 200 running words of the theme; this is a measure of the amount of repetitiveness, or (the scaling being in the opposite direction) of the diversity of vocabulary (2).

Grammar (G-19) is identical with the test used by Thurstone (11, test no. 57) except for a change in time limit.

Verbal Analogies (G-20) is virtually identical with Thurstone's test (11, test no. 41).

Spelling (G-21) is a list dictation test of spelling ability.

Suffixes (G-22), prepared by the writer, is modeled after a Suffixes test devised by Thurstone (15) which required the subjects to give all the words ending in the suffix -able which they could recall in the time allowed. The writer decided to use the suffix -en for the pres-sent test with the intention of making it sufficiently difficult for a college population.

Anagrams (G-23) is similar to the Anagrams test employed by Thurstone (11, test no. 15) except that the word OCCUPATION was substituted for PERVERSENESS, the test word used by Thurstone.

Rhyming (G-24). A test of rhyming ability employed by Thurstone (15) required the subjects to give four rhymes each to a list of some twenty words. For a college population, such a test seemed too easy. The writer therefore required the subjects to give as many rhymes as possible in a minute to each of a set of four words, graded in difficulty on the basis of the number of rhymes which several preliminary subjects were able to give.

Disarranged Words I (G-25) is identical with the Disarranged Words test used by Thurstone (11, test no. 12) except that it is shorter than the original test, only 7 of the 12 words in each meaning category being used. The time limit was set at 4 minutes.

Inventive Opposites (G-26) is virtually identical with Thurstone's test (11, test no. 10).

Phrase Completion (G-27) was devised by the writer in order to measure the extent to which individuals tend to conform to the lin guistic norm. The subjects are asked to complete items such as the fol lowing with the first word that comes to mind: "Hounds and

"And what do you.... " "But it's all. ." In a prelimi nary study, a test composed of approximately 75 items was adminis tered to several classes in psychology at the University of Minnesota. Frequency distributions were made of the responses to each item, and on the basis of these a scoring system was devised to measure the "community of response." Thus, in general, a credit of 3 was given to the most frequent response; 2 to the next most frequent; 1 to the third most frequent; and 0 to a response which did not appear com- 
monly in the responses of the population studied. An item analysis which was then carried out yielded 24 items which had sufficient discrimination power, on the basis of the total test score, to justify their inclusion in the present test. The test does not have a time limit.

The Speech Attitude Scale (G-28) is a published self-rating scale devised by Knower (6) to measure confidence and poise in speech situations. It was included in the present battery in order to see whether any of the primary mental abilities are associated with confidence in speaking.

Handwriting (G-29), devised by the writer, is presumably a measure of normal speed of handwriting. The subjects are required to copy a paragraph in blanks which are provided between the lines of the text. The score is a function of the number of letters written in 110 seconds. The test was included in order to provide a statistical control of speed of handwriting in the case of tests like G-15, G-32, and G-37 where speed of handwriting may be involved.

Morpheme Recognition (G-30, G-31) has been completely described in a previous publication of the writer (3). It was originally devised as a test of the ability to recognize the meanings of roots, suffixes, and prefixes of Latin or Greek origin in the English language. Two scores are derived from the test: G-30 is derived from the responses in the left-hand parts of the items (Examples), and G-31 is the number of correct responses in the righthand parts of the items (Meanings). In the present battery, this test was a time-limit test.

The Letter-Star Test (G-32, G-33) had been devised by the writer several years before the planning of this investigation in connection with the problem of the mathematical theory of word-frequency distribution (2). In this test, the subject is presented with patterns of letters and asterisks such as $* \mathrm{Y} * \mathrm{~S}$. He is to respond by substituting a word of his own choice for each symbol in the pattern, with the sole restriction that words substituted for capital letters must begin with the letter indicated. A sample response for * $\mathrm{Y} * \mathrm{~S}$ might be Is your father sick? In the construction of the test, the frequencies with which the various letters appear were determined according to the frequency distribution of initial letters in English; some adjustment was made, however, for the initial letters of the most common words; e.g., for $\mathrm{T}$ in the, 0 in of. The two scores which were derived from the subjects' responses in the present investigation are: G-32, the number of items completed in 10 minutes, and G-33, the number of different words in the first 100 running words of the responses.

Paired Associates (G-34, G-35) was devised by the writer as a test of the $M$ factor, with special reference to the way in which the memory factor might be expected to be important in learning foreign 
languages. In the practice period, the subject is required to memorize a vocabulary of Turkish words with their English meanings. On the two test pages, he is asked first to give the English meanings of the Turkish words and then to give the Turkish equivalents of the English words. Separate scores (G-34, G-35) are derived from each of the test pages. The writer's test differs from Thurstone's memory tests with respect to the way in which the subject is given opportunity to learn the material to be memorized. Thurstone, in most cases, has merely required the subjects to reproduce the associations once during learning and then to study silently until time is called. The writer, reversing this procedure, required two minutes of intensive study of the associations before preliminary written practice of the associations was attempted. It was believed that in this way closer attention would be paid to the material and that the preliminary practice would aid learning to a greater extent. This procedure was also devised in order to minimize individual differences in ability to organize the learning.

Vocabulary (G-36), devised by the writer, is similar to current multiple-choice vocabulary tests. It was believed that the present test would prove to have a more desirable score distribution, range of difficulty, and sensitivity than either of the tests employed by Thurstone (11, tests no. 58 and no. 60).

Distorted English (G-37) was constructed in an attempt to measure the ability to perceive meaning in foreign language idiom. It is the experience of foreign language teachers that students often have difficulty in assembling a number of isolated and apparently disarranged meaningelements into a larger meaningful whole. One way in which such an ability might be measured would be to ask the subject to make an idiomatic rendering of sentences in French or German translated word-for-word into English. In order to control the factor of previous foreign-language experience, the writer used literal translations of passages in more exotic languages - namely, Hungarian and various American Indian languages. In the scoring, which was made as objective as possible, credits are given for the correct rendition of certain features in the literal translation. The test was administered as a time-limit test, but it later appeared that this was unfortunate, since there were large differences in readiness to guess, exhibited, for example, by excessive slowness on the part of some subjects who were at the same time more accurate in their responses than speedier subjects.

Word-Choice (G-38), assembled by the writer, is in form somewhat similar to Grammar (G-19). Most of the items concern pairs of words which are commonly confused, such as derisive and derisory. 
Memory I (G-39) is identical with Thurstone's Word-Number memory test (11, test no. 46), except that the second fore-exercise has been omitted.

Disarranged Morphemes (G-40), devised by the writer, was included in order to test the hypothesis that the $W$ factor involves the ability to arrange various linguistic units in meaningful order. In contrast with tests which involve the rearrangement of letters into words, this test involves the rearrangement of syllables (morphemes) into two-word phrases. A sample item is:

-s quire ex ing re act ment

The subject is asked to rearrange these elements into two long words, an adjective and a noun (exacting requirements).

Similes (G-41) is identical with that used by Stumberg (8) in a study of poetic ability. The subjects are asked to give as many suggestions as possible for completing lines of poetry which require the use of simile. The score is simply the number of responses given, regardless of quality. The subjects were allowed 2 minutes for each of the 4 items of the test.

Normal speed of oral reading (1-42). The subject is required to read aloud a prose paragraph. The score is a function of the duration of reading in seconds.

Fastest speed of oral reading (I-43) is similar to I-42 except that the subject is asked to read aloud another paragraph as fast as possible without being unintelligible or inaccurate.

Naming states of the Union (1-44) is a test in which the subject is asked to name the states of the United States as fast as possible within the time-limit.

Giving first names (I-45) is similar to the preceding test except that the subject is asked to give, orally, all the first names, either boys' or girls', that he can think of.

Memory for homophones (I-46) is similar to a test used by Davis in studying differences in imagery type (5). The subject is allowed to view a word-square composed of sets of homophones (such as CENT, SCENT, SENT) for 10 seconds, after which he is asked to reproduce the wordsquare as accurately as possible from memory. The scoring technique is similar to that recommended by Davis.

Speed of articulation (I-47) is a measure of the speed with which the subject can pronounce certain consonants in a series such as papapapa. . (where $a$ represents a neutral vowel). The score is a function of the number of seconds taken to make forty articulations.

Auditory memory span (I-48) is similar to a test devised by Anderson (1), who reports that it is correlated with intelligence, achievement in foreign languages, and English usage. It is administered and 
scored like conventional digit-span tests, but the elements to be memorized are simple vowel sounds rather than digits.

Picture Description (I-49, I-50, I-51, I-52) requires the subject to respond to a picture orally and in his own words. The picture to which the subjects are asked to respond is the portrait of Cardinal Guevara, "The Cardinal Inquisitor," painted by the artist known as El Greco. The subject, after being told how to use the Dictaphone, is read a paragraph of standard instructions and allowed to view the picture. He is then given two minutes to consider what he is to say, after which he is required to speak into the Dictaphone as continuously as possible for two minutes, still viewing the picture. The following scores are obtained from typewritten transcriptions of the Dictaphone recordings: I-49 is the number of "relevant" words spoken during the two minutes. The "relevant" words are considered to be the words which the subject "meant" to say and which would remain if the speech response as a whole were to be edited and freed of hesitations, repetitions, rephrasings, "ah's" and "er's," and the like. I-50 is the ratio of the relevant words to the total number of words (both relevant and irrelevant). This is claimed to be essentially a measure of the coherence or continuity of the speech response and has been used previously by Stinchfield (7). I-51 is a composite rating, by expert judges, of the quality of the speech response as transcribed; in many respects it is similar to the rating of the themes (G-14). I-52 is a measure of diversity of vocabulary, the number of different words in the first 100 relevant words of the speech response.

Form-Naming (I-53) and Color-Naming (I-54) are tests which were originally devised by Woodworth and Wells (17). They were included here because they seemed to involve a type of facility in verbal association. The scores are functions of the time taken in naming the forms or colors.

Paragraph Memory (I-55) is taken from the Stanford-Binet intelligence scale $(9$, pp. 186,188$)$, and is scored by the method of retained members. After hearing a paragraph read by the administrator. the subject is asked to reproduce it orally from memory as accurately as possible.

Nonsense Numbers (I-56) was devised by the writer as a test of one aspect of the ability to learn and comprehend foreign languages as spoken. The subject is taught a simple artificial system of number expression utilizing nonsense syllables. This is analogous to teaching the number system of a foreign language. The subject is then asked to write down the arabic numeral equivalents of a list of numbers in the artificial system read aloud in a standard fashion by the experi- 
menter. The score is the number of digits correctly written on the answer blank.

The 42 tests were arranged in two group testing sessions of two hours each and one individual testing session of one hour.* The Speech Attitude Scale (G-28) was filled out by the subjects outside of the test periods at leisure.

The subjects were for the most part college undergraduates at the University of Minnesota who volunteered to take the tests on being promised individual reports of their standings. Although more than 170 individuals took at least some of the tests, only 119 cases were found to be complete. Of these 119 subjects, 57 were men and 62 were women. With respect to educational status, the subjects were distributed as follows: Freshmen, 28; Sophomores, 37; Juniors, 21; Seniors, 20; Graduate students, 9; Adults not in school, 2; Unknown, 2. A large number of the subjects were majoring or were planning to major in academic fields involving language, such as English composition, speech, foreign languages, and journalism. All subjects were native speakers of English, but there was found to be considerable variety in home language background. Data on the academic achievement and genera] scholastic aptitude of a considerable number of subjects were available at the University Testing Bureau. If we assume that these subjects are representative of the total group of 119 subjects, it can be concluded that the group was highly selected, since the means of our samples with respect to high school percentiles and college aptitude tests were significantly above the corresponding means for the liberal arts college population.

Before the scores on the 42 variables were used in computing the correlational matrix necessary in the factorial analysis, it was considered desirable to take two steps; namely, (1) normalization (where necessary) of the raw score distributions, and (2) coding of the score distributions in ten class intervals so that the data for a single case could be punched on a standard Hollerith card of 80 columns, each variable being represented by one column. With the exception of several studies in which a two-factor type of analysis was used, this is probably the first factorial study in which score distributions have been normalized. Thurstone, in his first large factorial study (11), used tetrachoric rather than product-moment correlation coefficients on the ground that the use of tetrachoric correlations automatically normalizes the underlying score distributions, thus satisfying one

* A micro-film copy of the test battery, including instructions and fore-exercises, is available for 80 as an Auxiliary Publication, Document 1597, of the American Documentation Institute, Offices of Science Service, 2101 Constitution Ave., Washington,
D. C. 
of the assumptions of multiple-factor analysis. He admitted, however, that "the most complete procedure would . . . seem to be to normalize each of the distributions of raw scores and then to compute the product-moment coefficients" (11, p. 58). In subsequent factorial studies of Thurstone, tetrachoric correlations were discarded in favor of product-moment correlations, since the former appeared to introduce an unreasonable amount of error variance, but in no case has the original suggestion of normalizing the distributions been carried out. In the present study it was decided to make reasonably sure that all score distributions involved in inter-correlations were normal. Quite apart from any considerations of the effect of distribution form on factorial structure, the assumptions underlying the product-moment correlation coefficient justify this step. The ultimate justification for the normalization of score distributions is the assumption that mental abilities are in reality distributed normally and that the deviation of a distribution from normality is a function of the specific character of a test, the conditions under which it is administered, the scoring technique, or the sampling of subjects. Many of the raw score distributions were surmised to be normal, at least with respect to skewness, merely by inspection; no rigorous test was applied to these distributions because of the labor which would have been involved. All distributions which appeared suspiciously nonnormal were tested for normality by R. A. Fisher's $g$ statistics; as it happened, all these distributions were found to be skewed and in many cases nonmesokurtic. The distributions which were found to deviate from normality were transformed by various functions until the statistical test left little doubt that they were normal.

The product-moment intercorrelations, presented in Table 1, were computed from sums of squares and cross-products obtained by Hollerithmachine procedures. The values were not corrected for grouping or for attenuation. The coefficients are for the most part positive in sign, the largest negative coefficient in the table being -.251. Variables G-16, I-48, and I-52 were eliminated from the correlational matrix used in the factor analysis because they were seen to have little correlation with other variables. The correlations of tests G-30 and G-31 with other tests are not used in the final correlational matrix. These tests had such a high correlation with each other $(\mathrm{r}=.888$, or 1.013 when corrected for attenuation) that it was deemed advisable to combine them into a new variable, G-30a. All correlations with G-30a are computed on the basis of the sums of the paired coded scores on variables G-30 and G-31. A similar procedure might have been employed in the case of tests G-34 and G-35, which were also highly correlated $(r=.835$, or 1.016 when corrected for at- 
tenuation), but for the sake of experiment it was decided to leave these scores separate in order to see how the factorial structure would be affected.

After the changes described in the preceding section had been made, a correlational matrix of 38 variables remained to be analysed by the multiple-factor analysis of Thurstone (10). The first step in this procedure was to find the centroid matrix of factor loadings on arbitrary co-ordinate axes. This is presented in Table 2. Ten factors were extracted from the correlational matrix; no more factors were taken out since the tenth factor residuals seemed small enough, when all criteria which were then available were considered, to indicate that little common-factor variance remained in the residual table. As will be seen, only nine factors could subsequently be rotated to simple structure, the tenth factor being made a residual plane. On the basis of a criterion recently developed by Coombs for determining the presence of significant common factor variance in a residual table (4), it has been found by the writer that it would have been profitable to have extracted another factor or possibly several factors after the tenth centroid factor in order to obtain a more convincing structure than the one reported in this paper. Nevertheless, according to Coombs the presence of one residual plane in the rotated factorial structure insures that enough centroid factors have been extracted to justify the psychological interpretation of the primary factors obtained.

Table 2 also presents the communalities $\left(h^{2}\right)$ of the test variables, values which indicate the proportion of variance in the test scores which is accounted for by the ten common factors extracted.

The second and final step in the factorial analysis was the rotation of the arbitrary orthogonal axes to the primary axes of a simple structure. The rotation of the present centroid matrix was accomplished partly by the method of extended vectors (13). Use was also made of certain other procedures which have not as yet been fully described in the literature. It will suffice to say that a theory of correlated primary factors developed by Tucker (16) underlies many of the methods employed by the writer.

It was possible to rotate 9 dimensions into simple structure, the 10th dimension remaining on a residual plane not subject to psychological interpretation. The transformation matrix, which in the present case was obtained after 17 rotations, is shown in Table 8, and the final rotated factorial matrix, consisting of projections of the test vectors on primary

planes, is presented in Table 4. The cosines of the angles between the reference vectors underlying the final projections are shown in Table 5. It should be inferred from this table that sim- 
ple structure demanded other than strictly orthogonal reference vectors. As a result, the correlations of the primary factors in many cases deviated from zero to an appreciable extent; these correlations are shown in Table 6. The matrices of Table 6 have been factored to obtain the saturations of the primaries in a second-order general factor according to a formula originally developed by Spearman and modified by Thurstone (10, p. 146). These saturations are given in Table 7.

The practice of designating primary factors by letters or symbols which are intended to suggest the nature of the corresponding abilities (or the corresponding general traits, in the case of factor studies of personality) is objectionable, in the opinion of the writer. A certain factor, for example, which Thurstone found in a series of studies and interpreted as a verbal factor, has been called $V$, but the present study appears to have broken up this factor into several factors. With the rapid strides in factorial research it is becoming apparent that the convenience of such a practice is illusory. Until the ultimate unities of ability have been isolated and interpreted in a definitive manner, it seems prudent to designate the factors in each study by purely arbitrary tags. This paragraph will serve to explain the writer's practice.

The location of the primary trait vectors designated as $C$ and $J$ presented the only serious problem in the process of rotation. The crux of the difficulty was whether $C$ and $J$ could best be regarded as correlated or as uncorrelated primary factors. It was found that when the reference vectors $C$ and $J$ were rotated into simple structure, they were highly oblique, the cosine of their angular separation being -.40, a figure connoting a substantial positive correlation of the corresponding primaries. Nevertheless, there seemed to be a fair likelihood that a corner of the configuration was missing, and that the factors $C$ and $J$ were not to be regarded as correlated. Factor $C$ was thought to be similar to the verbal factor $V$ previously identified by Thurstone, and factor $J$ was taken to be some sort of reasoning factor. Inasmuch as the tests having high projections on the $J$ plane included verbal material which would be expected to result in appreciable loadings on the $C$ factor, the reference vectors were set orthogonal, with the result that the tests of the primary $J$ were given appreciable projections in the general dimension of $C$. This rotation resulted in a new set of projections for all the tests in this dimension; this new set of projections, for the uncorrelated case, is given in column $C$ of Table 4. In any event, the problem of rotation discussed here is not crucial in the interpretation of the factors involved.

Inspection of the rotated factorial matrix (Table 4) reveals that. 
in the main a positive manifold has been obtained, i.e., that most of the appreciable projections are positive and that few of the negative projections deviate substantially from zero. This is the usual result in the factor analysis of mental abilities. Only two negative projections appear to be significantly different from zero; namely, that of test G-28 on $C$ or $\mathrm{C}^{\prime}$ and that of test G83 on $H$.

We may now consider the interpretations of the factors. Projections of .30 or greater will for convenience be regarded as significant for the purposes of interpretation.

One of the clearest factors identified in the present study is the $C$ or $C$ factor. The tests which have projections on $C$ and $C$ which can be regarded as significant are listed below, together with their significant loadings on other factors.

\begin{tabular}{|c|c|c|c|c|}
\hline \multicolumn{5}{|l|}{ Code } \\
\hline No. & Test & $\mathrm{C}$ & $C^{\prime}$ & Other projections \\
\hline G-38 & Word-Choice & .52 & .64 & - - \\
\hline G-36 & Vocabulary & .43 & .55 & $g(.37)$ \\
\hline $\mathrm{G}=27$ & Phrage Conpletion & .47 & .52 & $H(.39)$ \\
\hline$G+19$ & Grammar & .44 & .49 & $E(.38)$ \\
\hline$I-46$ & Memcsy for homophones & .43 & .48 & - \\
\hline$G-24$ & Rhyming & .46 & .47 & $\ldots$ \\
\hline $\mathrm{G}-21$ & Sjęling & .40 & .44 & $D(-1 i)$ \\
\hline $\mathrm{G}+30 \mathrm{~s}$ & Morpheme Jecognition & $(.21)$ & .42 & $J(.+1\}$ \\
\hline $\mathrm{G}-40$ & Disarranged Morphemes & $(.23)$ & .42 & $J(., 78)$ \\
\hline$G=14$ & Therue-Rating & .99 & $.4 !$ & $G(.39)$ \\
\hline G-13 & Fisarranged Words II & $(.23)$ & .97 & 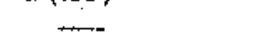 \\
\hline $1-55$ & Partalaph Memory & $(25)$ & .35 & $F(\therefore 9)$ \\
\hline $\mathrm{G}+37$ & Distorted Engtish & +30 & .84 & $E(2.) ; G(.48)$ \\
\hline G-2.2 & Suftixes & $(.27)$ & .32 & $A(55)$ \\
\hline G-28 & Spech Attitude Scale & -+36 & -41 & - \\
\hline
\end{tabular}

It is fairly obvious that the tests which have appreciable positive loadings on this factor involve some sort of intellectual verbal ability, It is to be noted that two tests which have high projections in the list above (G36, G-19) are similar to corresponding tests in Thurstone's study (11) which had high projections on what was designated as the $V$ (Verbal Relations) factor. On the basis of the factorial composition of these and similar tests in the list given above, the present $C$ factor can with considerable confidence be closely identified with the previously discovered $V$ factor. Nevertheless, it sometimes happens that for various reasons a factor in one investigation is resolved or split into two or conceivably more than two factors in subsequent investigations. In this way, it may be conceived that a subsequent investigation may sample only one of several sub-factors underlying a single factor in a previous study. It is therefore quite possible that 
the $C$ tests in the present battery have sampled only one or several constituent factors in what Thurstone has quite justifiably regarded as a single factor, on the basis of his data. Whatever the case may be, it can at least be said that the present $C$ factor has something in common with the $V$ factors found in previous factorial studies. It is to be carefully noted, however, that two tests of Thurstone's $V$, Inventive Opposites (G-26) and Verbal Analogies (G-20), do not appear on the present $C$ factor. The issues raised by this fact will be discussed subsequently.

Merely to say that the present $C$ factor involves some sort of intellectual verbal ability is unsatisfactory. Tests exist in the battery which can also be regarded as involving intellectual verbal ability but which do not have significant projections on $C$ or $\mathrm{C}^{\prime}$.

Close examination of the data available leads the writer to conclude tentatively that this factor represents the individual differences in some aspect of the ability to learn various conventional linguistic responses and to retain them over long periods of time. The factor represents differences in the stock of linguistic responses possessed by the individual - the wealth of the individual's past experience and training in the English language. By conventional linguistic response may be understood any fact of speech behavior which is essentially arbitrary but which occurs with a certain frequency in definite situations. A response (e.g., the response underlying a phoneme) may not even have any intrinsic semantic value, though most linguistic responses do have such a value. The concept of conventional linguistic response described here is exemplified by words and meanings of words; phonological, morphological, and syntactical features of the language; certain expressive gestures; and patterns of idiomatic expression. (The writer assumes that formal characteristics of a language correspond in some way to responses in a psychological sense.)

Many tests of the $C$ factor listed above can be regarded as tests of the presence or absence of certain conventional linguistic responses under certain stimulus conditions. Grammar (G-19) tests the presence (either by recognition or recall) of certain morphological and syntactical responses. Several tests involve the size of vocabulary, such as Word-Choice (G-38), Vocabulary (G-36), Spelling (G-21) (since a number of infrequent words were included in the test), Morpheme Recognition (G-30a), Disarranged Morphemes (G-40), and possibly Rhyming (G-24) and Suffixes (G-22), if it is considered that individuals possessing large vocabularies are at an advantage in these latter tests. Phrase Completion (G-27) tests the presence of certain conventionalized patterns of expression, which, although utilizing rather common words for the most part, are themselves used with 
varying frequency and which have many of the characteristics of conventional linguistic responses as described here. Test G-14 (ThemeRating) can easily be regarded as involving the richness of the subject's stock of linguistic responses, particularly those characteristic of standard or accepted speech.

The interpretation of the $C$ factor made in the preceding paragraph does not apply so obviously to the remainder of the tests listed above. Memory for Homophones (I-46) has a fairly high saturation on $C$ and no other appreciable projections. In the light of as yet unpublished studies on memory abilities conducted by Thurstone, the writer believes that in the present battery the memory element in this test remains in its specific variance inasmuch as the particular type of memory ability involved is not tapped by any other test in the battery. If this is the case, the common factor variance of this test is not to be related to its memory element but to some other element, most probably to its verbal nature, since it utilizes pairs or triplets of homophones such as CENT-SENT-SCENT. The individual's knowledge of homophones acquired in past linguistic experience would probably be of service in performing this test, and such knowledge might possibly be drawn from the stock of linguistic responses which, according to the hypothesis maintained here, is represented by the $C$ factor. Disarranged Words II (G-13) has an appreciable saturation with $C$, but, contrary to expectation, no very remarkable projection on either of the factors which, as claimed below, are related to Thurstone's factor $W$. Most of the common factor variance in the test appears to be covered by the $C$ factor. This result becomes more plausible if it is recalled that the test was constructed with words of decreasing frequency of occurrence. The appearance of Paragraph Memory (I-55) among the $C$ tests may be interpreted as due to the relatively difficult vocabulary in the test paragraphs. The small but appreciable saturation of Distorted English (G-37) may be accounted for by the possibility that this test involves a knowledge of grammatical patterns.

We may now ask why Inventive Opposites (G-26) and Verbal Analogies (G-20), tests which Thurstone found to have appreciable projections on his $V$ factor, do not appear among the $C$ tests in the present factorial structure. It may be noted that neither of these tests, at least for the college population of subjects used here, can easily be regarded as involving individual differences in extent of vocabulary or wealth of linguistic responses. The words used in Verbal Analogies are common, and the factor making for variation in performance appears to be some sort of reasoning ability rather than knowledge of linguistic responses. Nor does extent of vocabulary appear to be high- 
ly important in Inventive Opposites, where the score is merely the number of words written, without regard to their adequacy in reference to the task set. The subject is likely to give any response which he thinks may be acceptable. If only correct responses were scored, or if the test were constructed in multiple-choice form with initial letters of possible answers given (as has been done in the machine-scored form of the test issued by the American Council on Education), there would be a substantial probability that the test would measure the ability represented by the $C$ factor of the present study. This would also be the case if the test were administered to school children not familiar with some of the words used in the test.

The writer is inclined to believe that Thurstone's $V$ factor is represented in the present investigation by two or possibly three component factors, $C$, $J$, and possibly $G$. This belief is supported by the fact that several tests which in the light of Thurstone's findings were expected to be pure $C$ tests actually appear in other factors. This was true of Verbal Analogies (G-20), which appears in $J$, and of Inventive Opposites (G-26) and Theme-Rating (G-14), which appear in G. Furthermore, this belief seems to be compatible with the interpretations of these factors which are offered. It would be fairly easy to design an experiment to yield further information on this point. It seems fairly clear, at least to the writer, that the present $C$ factor does not directly involve the manipulation of ideas or relationships but merely represents the knowledge of verbal tokens which underlies the manipulation of ideas and relationships. If anything, Thurstone's description of the factor $V$ seems to apply to the factor $J$ of the present study rather than to the factor $C$. Moreover, on the basis of the saturations of factors $C$ and $\mathrm{Z}$ o $\mathrm{n}$ a secondorder general factor (Table 7), factor $J$ appears to behave like Thurstone's factor $V$ (which according to a recent study [14] seems to have a high saturation on a general factor) more than does factor $C$.

The substantial negative projection of the Speech Attitude Scale (G-28) on $C$ is of interest. A tentative hypothesis useful in accounting for this result is that many individuals who have large stocks of linguistic responses are, so to speak, embarrassed by trop de richesse and have difficulty in selecting the most effective responses in a given situation. Persons of average verbal ability, on the other hand, rarely stop to choose words carefully. It is important to note, however, that the $C$ factor is negatively related only to confidence in speaking ability and not to actual speaking ability as presumably measured by tests I-49, I-50, and I-51.

The three tests which have substantial projections on the factor $J$ are Verbal Analogies (.54), Morpheme Recognition (.41), and Dis- 
arranged Morphemes (.38). The common element in these tests seems to be some sort of reasoning ability or ability to handle verbal relationships. Verbal Analogies (G-20) seems to be a pure test of $J$, having no appreciable projection on $C$, although previous results would lead one to expect it to have a substantial projection on $C$. As has been suggested above, factor $J$ appears to conform to the interpretation of the factor $V$ offered by Thurstone-namely, that the factor $V$ involves the manipulation of ideas. The writer is of the opinion that some of Thurstone's $V$ tests such as Disarranged Sentences, Verbal Classification, and Word-Grouping (11) would have appeared on factor $J$ if they had been included in the present battery.

The factor $G$ seems to be one of the most difficult to interpret on the basis of the present data. The author has not been able to arrive at any interpretation of the factor which can satisfactorily account for all the tests with appreciable projections on it. These tests are: Picture DescriptionRating (.53), Distorted English (.48), Similes (.44), Theme-Rating (.39), Maximum Speed of Oral Reading (.37), Inventive Opposites (.37), Speed of Handwriting (.37), Vocabulary (.37), Normal Speed of Oral Reading (.32), Picture Description - No. of relevant words (.32), and Letter-Star-No. of responses (.30). It has been suggested above that this factor (in its present state) is a component of Thurstone's original $V$ factor; it is further possible that this factor represents at bottom two or more separate factors which future investigation may reveal and that pure tests of these factors are missing in the present study. The only pure test of $G$ is apparently Speed of Handwriting (G-29), but it is difficult to conceive a connection between this test and Picture Description (I-51). It can readily be seen, however, that many of the tests involve handwriting speed - for example, Distorted English, Similes, Theme-Rating and Inventive Opposites. Therefore, one component of the factor $G$ may be handwriting speed. Until further investigation is made of the tests which appear on $G$ in the present configuration, the writer will not attempt to interpret this factor.

The tests with significant projections on $A$ are as follows: Suffixes (.55), Form-Naming (.41), Disarranged Words I (.38), Word-Number Memory (.38), Color-Naming (.33), and possibly Giving First Names (.28). During the rotation of the axes this factor appeared to be connected with Thurstone's $W$ factor on account of the presence of Suffixes (G-22) and Disarranged Words (G-25) among its tests. Further consideration of the data leads the writer to conclude, however, that this is not identical with Thurstone's $W$ factor but that it is probably one component of it. The present investigation appears to have divided the original $W$ factor into two constituent unities, fac- 
tors $A$ and $E$. Looking for an underlying unity in the tests listed above, we arrive at the hypothesis that this $A$ factor involves the speed of word association (usually for common words) where there is some element of restriction in the task imposed; i.e., where only one or a certain number of responses from the total reserve are correct. In Suffixes (G-22) and in Disarranged Words (G-25), for example, the test materials undoubtedly give rise to a number of implicit responses from which the subject must select the correct or acceptable responses. In the performance of Form-Naming (I53) and Color-Naming (I-54), a similar process appears to be necessary to some extent, for in each of these tests five responses have very high and probably equal strengths, but the subject must select the appropriate response for each successive stimulus. In Giving First Names (I-44) it can be conceived that the subject must select appropriate responses from the reserve consisting of personal names, names identical with those previously given by the subject, and other names. The only test whose projection on $A$ cannot be readily explained is Word-Number Memory (G-39), which correlates fairly highly with other $A$ tests in the battery (see the correlational matrix, Table 1.)

The tests which have appreciable loadings on the factor $E$ are ThemeNo. of words (.45), Grammar (.38), Similes (.36), Picture Description-Per cent of relevant words (.35), Distorted English (.33), and Anagrams (.31). Because of the presence of several tests which were formerly thought to be $W$ tests, namely, Grammar (G-19) and Anagrams (G-23), it is believed that this is one component of the $W$ complex discovered in previous investigations by Thurstone and others. Most of the $E$ tests involve in some way the rate of production for meaningful and syntactically coherent discourse where there is little restriction to definite responses. The highest projection is that of Theme-Word Count (G-15), which clearly involves facility in producing sentences which are sufficiently meaningful to be accepted by the subject. A superficially comparable measure, Picture Description - No. of relevant words (I-49), does not appear in the above list because, it is believed, it is not directly a measure of coherence, but only a measure of the amount which the subject had to say. Picture DescriptionPer cent of relevant words (I-50) appears among the $E$ tests because it is a fairly direct measure of coherence. Grammar (G-19) possibly involves an element of syntactical coherence, and the appearance of Similes (G-41) among these tests appears to be consistent with our hypothesis, although it seems to emphasize semantic rather than syntactical coherence. Distorted English (G-37) can be conceived as involving facility in bringing about syntactical and semantic coherence; in one sense Distorted English is a test of the 
ability to organize implicit verbal behavior (generally thought to be somewhat formless or chaotic) into explicit verbal behavior which is acceptable as formal speech. We cannot explain the appearance of Anagrams (G-23) among these tests, but its projection is probably too small to cause much dismay.

The tests with appreciable saturations in the factor $H$ are Color-Naming (.49), Letter-Star Test-No. of responses (.42), Giving First Names (.41), Form-Naming (.41), Phrase Completion (.33), Naming States of the Union (.29), and Letter-Star Test-Diversity ( -.42$)$. The common characteristic of these tests is what may be described as readiness in attaching an appropriate name or tag to a stimulus (even if it is only an arbitrary name, as in the case of the Letter-Star test). In the case of tests I-44 and I-45 (Naming States of the Union and Giving First Names, respectively), the stimulus is implicit and may reside in the subject's imagery. The negative loading of test G-33 on $H$ is a result of the fact that tests G-32 and G-33 are negatively correlated $(r=-.251)$, it being inferred that the subjects who are speedier in producing responses have more tendency to repeat responses and thus to use fewer different words. It should not be concluded that there is direct inhibition between $H$ and test G-33, however.

The four tests which have substantial projections on the factor $F$ are Picture Description-No. of relevant words (.61), Picture Description-Per cent of relevant words (.58), Picture Description-Rating (.55), and Paragraph Memory (.39). This factor may in the first instance be regarded as 'speaking ability, or ability to give spontaneous oral expression to one's ideas in an effective and coherent manner. Alternatively, the factor may be interpreted as involving the subject's ease and confidence in the specific experimental situation, a situation complicated by the presence of slightly discomforting apparatus (i.e., the Dictaphone). All the tests in the list above permit either interpretation, including Paragraph Memory (I-55), which involves an oral response somewhat similar to the responses required in the Picture Description test. Nevertheless, the fact that the Speech Attitude Scale (G-28) did not appear here seems to contradict, to some extent, the second of the alternative hypotheses, inasmuch as this test is presumed to measure almost precisely the kind of ease and confidence which is thought to be demanded in this experimental situation.

The factor I? is represented by three tests, Paired AssociatesTurkish-English (.79), Paired Associates-English-Turkish (.77), and Word-Number Memory (.41). This factor is easily seen to be similar to the rote learning factor $M$ isolated in previous factoral investigations. 
Column $D$ of the rotated factorial matrix has appreciable saturations in seven tests, as follows: Maximum Speed of Oral Reading (.67), Normal Speed of Oral Reading (.62), Speed of Articulation (.57), Spelling (.41), Letter-Star Test-Diversity (.37), Color-Naming (.36), and Form-Naming (.29). The interpretation that this factor represents motor skill in speech is obvious, and is based primarily on consideration of the characteristics of the first three of these tests, but the remaining tests seem to have elements on the basis of which they may be reasonably subsumed under the factor $D$. In the case of Spelling (G-21), there is a suggestion that spelling ability is associated with motor skill in pronouncing words. A fairly plausible interpretation of the appearance here of the Letter-Star Test-Diversity (G33 ) is that since the test involves the initial letters of words, general facility in articulation and in word pronunciation provides the subject with a greater range of responses which can be utilized in this situation. It is obvious, finally, that Color-Naming (I-54) and Form-Naming (I-53) involve speed of articulation.

The finding of a generalized speed of articulation factor should be of interest to workers in the field of motor abilities, in view of the highly specific character of most types of motor ability. It is fairly safe to conclude that the present articulation factor is generalized over at least several fairly distinct speech movements. The writer has computed correlations between the speed measurements of the three speech movements in pronouncing the stops $p, t$, and $k$, utilized in the Speed of Articulation test. These correlations are: $p$ and $t, r=.915 ; p$ and $k, r=.900, t$ and $k, r-.912$.

The factor designated $K$ is represented by a residual plane and is not subject to psychological interpretation.

The author is indebted to Professor L. L. Thurstone for generously allowing him to use the facilities at the University of Chicago for completing the factorial analysis. Special acknowledgment is due Mr. Ledyard R. Tucker for assistance in making the rotations from the centroid matrix. 
TABLE 1

The Inter-test Correlations* (119 cases)

\begin{tabular}{|c|c|c|c|c|c|c|c|c|c|c|c|}
\hline & 13 & 14 & 15 & 19 & 20 & 21 & 22 & 23 & 24 & 25 & 26 \\
\hline 13 & & .297 & .220 & .358 & .327 & .322 & .358 & .435 & .292 & .419 & .427 \\
\hline 14 & .297 & & .317 & .286 & .168 & .362 & .289 & .194 & .416 & .109 & .329 \\
\hline 15 & .220 & .317 & & .195 & .190 & .201 & .099 & .260 & .168 & .230 & .142 \\
\hline 19 & .858 & .286 & .195 & & .336 & .607 & .261 & .312 & .453 & .431 & .453 \\
\hline 20 & .327 & .168 & .190 & .336 & & .281 & .238 & .320 & .256 & .393 & .417 \\
\hline 21 & .322 & .362 & .201 & .607 & .281 & & .338 & .392 & .470 & .428 & .371 \\
\hline 22 & .358 & .289 & .099 & .261 & .238 & .338 & & .317 & .368 & .368 & .362 \\
\hline 23 & .435 & .194 & .260 & .312 & .320 & .392 & .317 & & .322 & .488 & .343 \\
\hline 24 & .292 & .416 & .168 & .453 & .256 & .470 & .368 & .322 & & .261 & .434 \\
\hline 25 & .419 & 109 & .280 & .431 & .393 & .428 & .368 & .488 & .261 & & .332 \\
\hline 26 & .427 & .329 & .142 & .458 & .417 & .371 & .362 & .343 & .434 & .332 & \\
\hline 27 & .129 & .200 & .136 & .280 & .174 & .270 & .180 & .174 & .258 & .216 & .250 \\
\hline 28 & -.131 & .042 & .054 & -.161 & -.005 & -.047 & .098 & -.041 & .041 & -.034 & .066 \\
\hline 29 & .212 & .210 & .308 & .213 & .109 & .202 & .182 & .273 & .229 & .251 & .206 \\
\hline $30 a$ & .379 & .247 & .238 & .529 & .545 & .498 & .271 & .352 & .380 & .375 & .370 \\
\hline 32 & .199 & .201 & .392 & .246 & .167 & .273 & .274 & .860 & .153 & .896 & .284 \\
\hline 33 & .036 & .203 & -.058 & .112 & .141 & .274 & .076 & -.016 & .180 & .058 & .127 \\
\hline 34 & .356 & .108 & -.026 & .268 & .264 & .165 & .087 & .230 & .158 & .252 & .278 \\
\hline 35 & .398 & .080 & -.097 & .278 & .292 & .243 & .120 & .286 & 190 & .260 & .317 \\
\hline 36 & .363 & .527 & .241 & .504 & .419 & .599 & .290 & .379 & .456 & .244 & .518 \\
\hline 37 & .287 & .331 & .426 & .358 & .194 & .305 & .264 & .350 & .375 & .215 & .426 \\
\hline 38 & .410 & .380 & .032 & .425 & .310 & .414 & .317 & .283 & .291 & .234 & .417 \\
\hline 39 & .189 & .005 & -.108 & .163 & .156 & .072 & .871 & .250 & .113 & .245 & .327 \\
\hline 40 & .478 & .307 & .310 & .493 & .480 & .488 & .459 & .454 & .443 & .441 & .517 \\
\hline 41 & .071 & .178 & .428 & .043 & .033 & .105 & .144 & .172 & .162 & .125 & 191 \\
\hline 42 & .128 & .309 & .286 & .187 & .229 & .278 & .200 & .108 & .265 & .202 & .194 \\
\hline 43 & .044 & .454 & .254 & .186 & .155 & .340 & .092 & .098 & .267 & .093 & .158 \\
\hline 44 & .087 & .048 & .111 & -.016 & .202 & .130 & .324 & .059 & .125 & .275 & .080 \\
\hline 45 & .039 & .071 & .227 & .112 & -.061 & -.047 & 107 & .096 & .094 & .145 & -.035 \\
\hline 46 & .303 & .103 & .037 & .248 & .108 & .260 & .260 & .158 & .418 & .208 & .161 \\
\hline 47 & -.072 & .095 & .133 & .012 & .115 & .144 & -.082 & -.063 & .040 & .054 & -.125 \\
\hline 49 & .009 & .264 & .378 & .034 & .032 & .006 & -.041 & .006 & .091 & -.031 & .154 \\
\hline 50 & .058 & .195 & .219 & .135 & -.022 & .140 & -.090 & .018 & .140 & -.026 & .056 \\
\hline 51 & .045 & .427 & .196 & .050 & .016 & -.105 & .051 & -.022 & .227 & -.062 & .237 \\
\hline 53 & .205 & .186 & .196 & .086 & .255 & .098 & .293 & .189 & .152 & .314 & .210 \\
\hline 54 & .172 & .154 & .264 & .082 & .150 & .284 & .256 & .248 & .142 & .409 & .188 \\
\hline 55 & .283 & .408 & .180 & .311 & .800 & .264 & .219 & .174 & .255 & .178 & .252 \\
\hline 56 & .398 & 090 & .126 & .317 & .310 & .234 & .359 & .370 & .300 & .396 & .292 \\
\hline 16 & .015 &,- 003 & .068 & -.029 & .051 & $-05 \overline{5}$ & .107 & -.075 & -.053 & $.05 \overline{5}$ & .010 \\
\hline 30 & .384 & .268 & .245 & .526 & .503 & .501 & .264 & .371 & .379 & .382 & .320 \\
\hline 31 & .341 & .211 & .216 & .501 & .559 & .404 & .262 & .310 & .358 & .344 & .402 \\
\hline 48 & .173 & -.050 & .007 & .091 & .124 & .053 & .170 & .148 & .198 & .067 & .205 \\
\hline 52 & -.045 & .114 & .006 & .104 & .093 & .088 & .109 & -127 & -.011 & .053 & .066 \\
\hline
\end{tabular}




\section{JOHN B. CARROLL}

TABLE 1 (continued) The

Inter-test Correlations

\begin{tabular}{|c|c|c|c|c|c|c|c|c|c|c|c|}
\hline & 27 & 28 & 29 & $30 a$ & 32 & 33 & 34 & 35 & 36 & 37 & 38 \\
\hline 13 & .129 & -131 & .212 & .379 & .199 & .036 & .356 & .398 & .368 & .287 & .410 \\
\hline & .200 & .042 & .210 & .247 & .201 & .203 & .108 & .080 & .527 & .331 & .380 \\
\hline 5 & .136 & .054 & .803 & .238 & .392 & -.058 & -.026 & -.097 & .241 & .426 & .032 \\
\hline 19 & .280 & -.161 & .213 & .529 & .246 & .112 & .268 & .278 & .504 & .358 & .425 \\
\hline 20 & .174 & -.005 & .109 & .545 & .167 & .141 & .264 & .292 & .419 & .194 & .310 \\
\hline 21 & .270 & -.047 & .202 & .498 & .275 & .274 & .165 & .243 & .599 & 305 & .414 \\
\hline 22 & .180 & .098 & .182 & .271 & .274 & .076 & .087 & .120 & .290 & .264 & .817 \\
\hline 28 & .174 & $-.04 t$ & .273 & .352 & .860 & -.016 & .230 & .286 & 379 & .350 & .288 \\
\hline 24 & .258 & .041 & .229 & .380 & .153 & .180 & .158 & .190 & .456 & .375 & 291 \\
\hline 25 & .216 & -.034 & .251 & .375 & .396 & .058 & .252 & .260 & .244 & .215 & .284 \\
\hline 26 & .250 & .066 & .206 & .370 & .284 & .127 & .278 &.$\$ 17$ & .618 & .426 & .417 \\
\hline 27 & & -.246 & .108 & .291 & .323 & -.125 & .096 & .070 & .403 & .352 & .380 \\
\hline 28 & -.246 & & .186 & -.042 & .026 & .134 & .089 & .033 & .068 & -.014 & -.187 \\
\hline 29 & .108 & .186 & & .147 & .312 & .016 & .114 & .060 & .282 & 399 & .132 \\
\hline $30 a$ & .291 & -042 & .147 & & .281 & -.020 & .801 & 270 & .697 & .381 & .442 \\
\hline 32 & .323 & .026 & .312 & .281 & & -251 & .090 & .001 & .306 & 355 & .137 \\
\hline 33 & -125 & .134 & .016 & -.020 & -.251 & & .022 & .055 & .095 & -.062 & .170 \\
\hline 34 & .096 & .089 & .114 & .301 & .090 & .022 & & .835 & .234 & .109 & .223 \\
\hline 35 & .070 & .033 & $.060^{\circ}$ & .270 & .001 & .055 & .835 & & .269 & .060 & .236 \\
\hline 36 & .403 & -.068 & .282 & .597 & .306 & .095 & .234 & .269 & & .477 & .564 \\
\hline 87 & .352 & -.014 & .399 & .381 & .836 & -.062 & .109 & .060 & .477 & & 267 \\
\hline 38 & .380 & -.187 & .132 & .442 & .137 & .170 & .223 & .236 & .564 & .267 & \\
\hline 39 & -.063 & .083 & .172 & .064 & .148 & .057 & .422 & .387 & .076 & .012 & .170 \\
\hline 40 & .266 & -.071 & .297 & .530 & .279 & .186 & .249 & .321 & 601 & .480 & .452 \\
\hline 41 & .027 & 201 & .235 & .152 & .374 & -.004 & .035 & -.017 & .161 & $405^{\circ}$ & -.096 \\
\hline 42 & -.084 & .185 & 379 & .184 & .265 & .284 & .126 & .111 & .812 & .245 & .118 \\
\hline 48 & .089 & .279 & .352 & .128 & .177 & .824 & .166 & .116 & .316 & 193 & .069 \\
\hline 44 & .184 & -151 & -.042 & .146 & .190 & .061 & .121 & .169 & .048 & .018 & .152 \\
\hline 45 & -.069 & -.053 & .010 & .033 & .198 & -.107 & -.007 & -.048 & -.128 & .066 & -.056 \\
\hline 46 & .262 & -.101 & .088 & .246 & .028 & .141 & .226 & .339 & .252 & .228 & .342 \\
\hline 47 & -.018 & .221 & .146 & .014 & .006 & .246 & .029 & -.009 & .058 & -.029 & -.158 \\
\hline 49 & .139 & .262 & .246 & .044 & .189 & .221 & .073 &.,- 079 & .143 & .112 & .119 \\
\hline 50 & -.020 & .254 & .044 & .026 & .101 & .188 & .048 & -.028 & .167 & .071 & .130 \\
\hline 51 & .010 & .211 & .181 & $-\$ 12$ & .200 & .221 & .014 & -.096 & .308 & .168 & .146 \\
\hline 53 & -.016 & .207 & .140 & .108 & .226 & .102 & .201 & .181 & .060 & .175 & .052 \\
\hline 54 & .121 & .115 & .307 & .108 & .311 & -.033 & .153 & .186 & .174 & .198 & -.034 \\
\hline 55 & .227 & .010 & .130 & .294 & .151 & .130 & .188 & .198 & .487 & .182 & .451 \\
\hline 56 & .103 & -.075 & .174 & .363 & .193 & .069 & .376 & 451 & .282 & .230 & .263 \\
\hline 16 & & .080 & .085 & Tus & .174 & .066 & -.075 & -.204 & -.047 & -.031 & .000 \\
\hline 30 & .284 & -.028 & .154 & $(.974)$ & .241 & -.010 & .281 & .266 & .574 & .363 & .410 \\
\hline 31 & .281 & -.054 & .131 & $(.969)$ & .307 & -.030 & .305 & .259 & .586 & .379 & 452 \\
\hline 48 & -.086 & .001 & .071 & .075 & .043 & .042 & .119 & .164 & .197 & .106. & -.031 \\
\hline 52 & .012 & .099 & .084 & .019 & .083 & -.023 & -.017 & -.122 & .076 & .082 & .112 \\
\hline
\end{tabular}


TABLE 1 (continued) The

Inter-test Correlations

\begin{tabular}{|c|c|c|c|c|c|c|c|c|c|c|c|}
\hline & 39 & 40 & 41 & 42 & 43 & 44 & 45 & 46 & 47 & 49 & 50 \\
\hline 13 & .189 & .478 & .071 & .123 & .044 & .087 & .089 & .803 & -.072 & .009 & .058 \\
\hline 14 & .005 & 907 & .178 & .909 & .454 & .018 & .071 & .103 & .095 & .264 & .195 \\
\hline 15 & -.108 & .310 & .428 & .286 & .254 & .111 & .227 & .037 & .189 & .378 & .219 \\
\hline 19 & .163 & .493 & .043 & .187 & .186 & -.016 & .112 & .948 & .012 & .034 & .135 \\
\hline 20 & .150 & 480 & $.03 \%$ & .229 & .155 & 202 & -.061 & .108 & .115 & .032 & -.022 \\
\hline 21 & .072 & .488 & .105 & .978 & .340 & .130 & -.047 & .260 & .144 & .006 & .140 \\
\hline 22 & .971 & .459 & .144 & .200 & .092 & .324 & 107 & .260 &,- 082 & -.041 & -.090 \\
\hline 29 & .250 & $.4 \breve{54}$ & .172 & .108 & .098 & 059 & .096 & .153 & -063 & .006 & .018 \\
\hline 24 & .113 & .443 & .162 & .265 & .267 & .125 & .094 & .418 & .040 & .091 & .140 \\
\hline 85 & 25 & .441 & .125 & .202 & .098 & .275 & .145 & .208 & .054 & -.031 &.- \\
\hline 26 & .527 & .517 & .191 & .194 & .158 & .080 & -.035 & .161 &,- 125 & .154 & .056 \\
\hline 27 & -068 & .200 & .027 & -.034 & .089 & .184 & -.069 & .262 & -.018 & .139 & -020 \\
\hline 28 & .083 & -.071 & .201 & .185 & .279 & .151 & -053 & -.101 & .221 & .262 & .254 \\
\hline 29 & .172 & .297 & .295 & .979 & .352 & $-.0 \div 2$ & .010 & .038 & .146 & 246 & .044 \\
\hline $30 a$ & .064 & .530 & .152 & .184 & .128 & +140 & .083 & .246 & .014 & .044 & .026 \\
\hline 32 & .148 & .279 & .374 & .260 & .177 & .190 & .198 & .028 & .006 & .189 & .101 \\
\hline 33 & .057 & .186 & -.004 & .284 & .324 & .061 & -.107 & .141 & .246 & .221 & .188 \\
\hline 34 & .422 & 249 & .085 & .196 & .166 & .121 & -.007 & .226 & .029 & .073 & .048 \\
\hline 35 & .387 & .321 & -.017 & .111 & .116 & .169 & -.048 & .339 & -.009 & -.079 & -.028 \\
\hline 6 & .076 & .601 & .161 & .312 & .316 & .048 & -.128 & .252 & .038 & .143 & .167 \\
\hline 87 & .012 & .480 & $.40 \overline{5}$ & 245 & .193 & .018 & .066 & .228 & -.029 & .112 & .071 \\
\hline 38 & .170 & .459 & -.000 & .118 & .069 & .152 & -.050 & 342 & -.158 & .119 & .130 \\
\hline 89 & & .292 & .034 & nis: & -.01 .6 & .077 & 074 & .033 & -.080 & .116 & -1.123 \\
\hline 40 & .202 & & .906 & .380 & .248 & .228 & -.003 & .293 & .107 & .059 & .086 \\
\hline 41 & .034 & .200 & & $.24 \pi$ & .268 & .148 & .398 & .056 & .118 & .326 & 127 \\
\hline 42 & .083 & .380 & .247 & & .675 & .041 & .104 & .143 & .421 & .237 & .085 \\
\hline 43 & -.016 & .248 & .268 & .675 & & .057 & .089 & -.002 & .475 & .342 & .180 \\
\hline 44 & .077 & .228 & .148 & .041 & .057 & & .172 & .120 & -.042 & .089 & .000 \\
\hline 45 & .074 & -.003 & .338 & .104 & .089 & .172 & & -.065 & .041 & .175 & .089 \\
\hline 46 & .033 & .293 & .056 & .143 & -0.02 & .120 & -.065 & & -.061 & .056 & .180 \\
\hline 17 & -.080 & .107 & .118 & .421 & .475 & -.042 & .041 & -061 & & .153 & .071 \\
\hline 19 & .116 & .059 & .326 & .287 & 342 & .089 & .175 & .056 & .158 & & .308 \\
\hline 50 & -.123 & .086 & .127 & .085 & .180 & .000 & .000 & .130 & .071 & .308 & \\
\hline 51 & -.038 & .153 & .192 & .228 & .890 & .048 & .089 & .017 & .138 & .558 & .885 \\
\hline 53 & .294 & .257 & .277 & .350 & .262 & .290 & .362 & T. & .183 & .150 & .020 \\
\hline 54 & .196 & .292 & .290 & .352 & .342 & .277 & .283 & .040 & .208 & .150 & .070 \\
\hline 5.5 & .180 & .340 & .086 & .216 & .195 & .034 & -103 & .218 & .060 & .249 & .289 \\
\hline 56 & $.3 L 5$ & .465 & .099 & .267 & .168 & .198 & .071 & .340 & .078 & .019 & .034 \\
\hline & .090 & .051 & -.027 & .104 & .014 & .157 & .024 & -.070 & .132 & .170 & -.078 \\
\hline & .089 & .514 & .142 & .185 & .196 & .111 & .002 & .257 & .029 & $.01 \overline{5}$ & .032 \\
\hline 31 & .087 & .516 & .154 & .173 & .112 & .175 & .066 & .219 & -.004 & .074 & .019 \\
\hline 48 & .062 & .166 & .050 & .170 & .063 & -.149 & -.028 & .210 & .082 & 019 & .090 \\
\hline 52 & -.030 & -109 & .910 & .087 & -.013 & .105 & -006 & -.192 & -.006 & .048 & -.019 \\
\hline
\end{tabular}




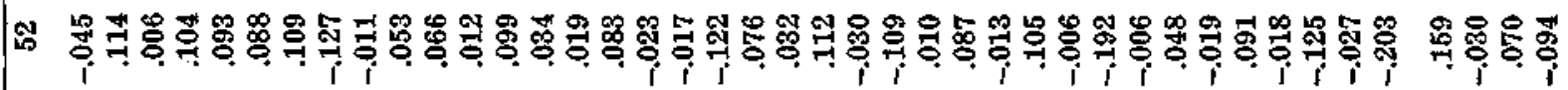

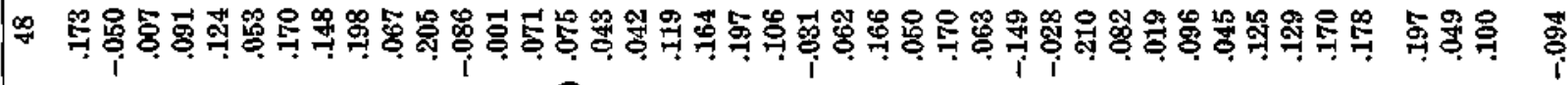

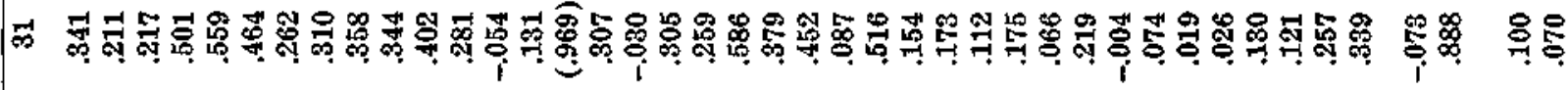

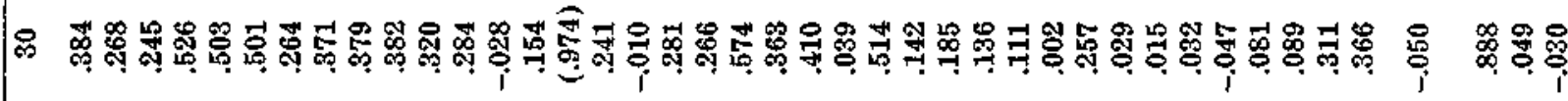

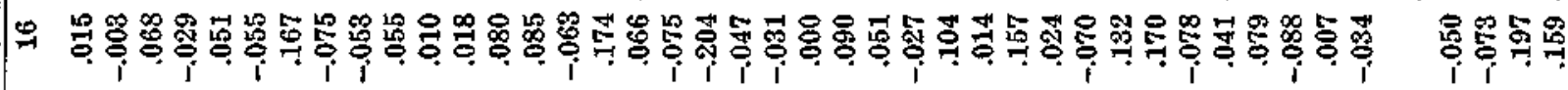

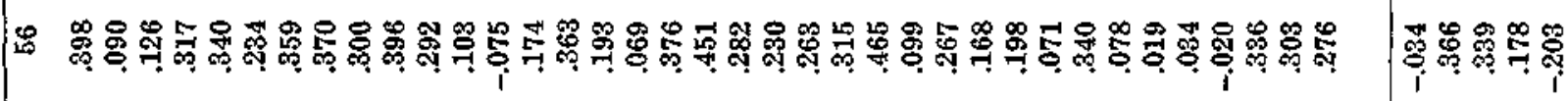

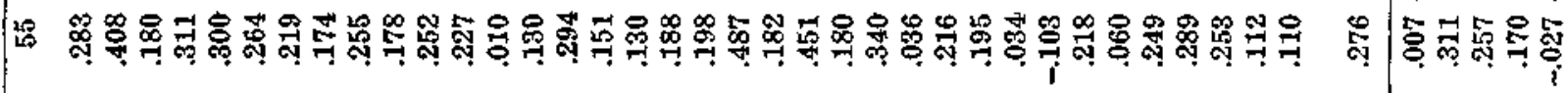

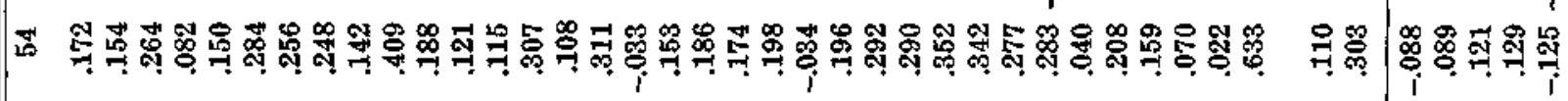

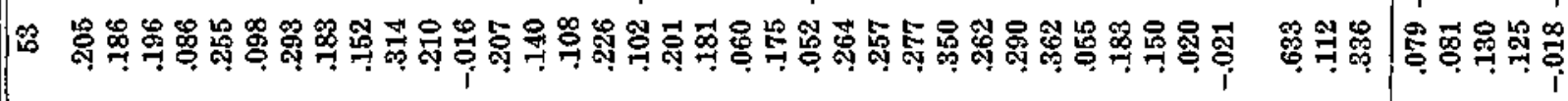

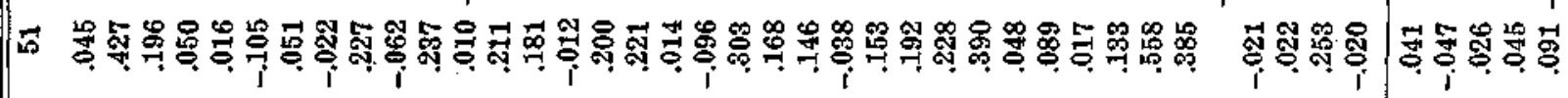

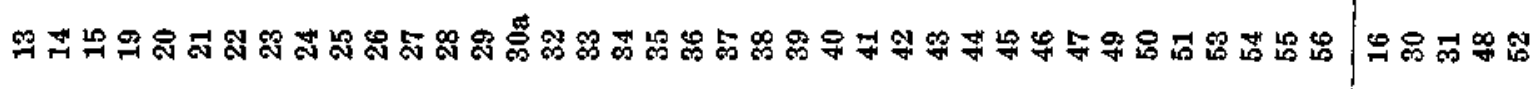


TABLE 2 The

Centroid Matrix

\begin{tabular}{|c|c|c|c|c|c|c|c|c|c|c|c|}
\hline & 1 & II & $I I I$ & $I V$ & $\boldsymbol{V}$ & $V I$ & $V I I$ & $V I I I$ & $1 x$ & $X$ & $h^{2}$ \\
\hline 13 & .531 & -.325 & .093 &,- 086 & -.093 & -.027 & -.030 & -.077 & -.098 & -.090 & 438 \\
\hline 14 & .538 & .127 & -.328 & -.075 & -166 & -.079 & .093 & .091 & .134 & -.089 & .494 \\
\hline 15 & .432 & .307 & -.056 & -348 & .166 & .046 & -.238 & -.095 & -.176 & -.074 & .537 \\
\hline 19 & .578 & -.939 & -218 & .041 & .199 & -.106 & -.133 & -.181 & .068 & .142 & .624 \\
\hline 20 & .506 & -.295 & .036 & .044 & .119 & .111 & .134 & .179 & -.329 & -.101 & .509 \\
\hline 21 & .606 & -.212 & -.211 & .076 & .249 & -212 & .110 & -.128 & .072 & .197 & .642 \\
\hline 22 & .504 & -.157 & .195 & -.128 & -.128 & -.222 & .316 & -.133 & .082 & -102 &, 533 \\
\hline 25 & .520 & -.241 & .182 & -.183 & .124 & $\quad .059$ & .051 & -.190 & -.099 & .105 & .473 \\
\hline 24 & .581 & -.134 & -206 & .040 & -.034 & -.142 & .048 & -.230 & .182 & -.168 & .586 \\
\hline 25 & .553 & -.175 & 307 & -.084 & .199 & -.167 & .064 & -.085 & -.164 & .159 & .569 \\
\hline 26 & .604 & -248 &,- 014 & -.093 & -.144 & .164 & .175 & -.108 & .090 & .054 & .534 \\
\hline 27 & .328 & -.239 & -206 & -.313 & .029 & -.088 & -.090 & .304 & .151 & .108 & .445 \\
\hline 28 & .112 & .408 & .099 & .211 & -.204 & .222 & .170 & -.147 & -.058 & .070 & .383 \\
\hline 29 & .431 & .149 & -.039 & -.115 & .139 & .231 & .086 & -.131 & .084 & .124 & .342 \\
\hline $30 \mathbf{a}$ & .582 & -.348 & -.076 & -070 & .221 & .084 & -.095 & .096 & -.168 & -.060 & .576 \\
\hline 32 & .468 & .113 & .147 & -.486 & .151 & .087 & -.056 & .047 & .053 & .216 & .529 \\
\hline 33 & .211 & .126 & -.954 & .428 & -.132 & -.126 & .278 & -.143 & -.172 & -.096 & .478 \\
\hline 34 & .443 & -.266 & .324 & .381 & -.170 & .325 & -.282 & .146 & .144 & .034 & .774 \\
\hline 35 & .429 & -.397 & .352 & .453 & -.145 & .299 & -.200 & .108 & .160 & -0055 & .827 \\
\hline 36 & .684 & -.246 & -.400 & -.119 & $\quad .042$ & .128 & .094 & .196 & .077 & .050 & -776 \\
\hline 37 & .535 & -.042 & -.172 & -359 & .150 & .143 & -.084 & -.189 & .185 & -1.37 & .588 \\
\hline 38 & .505 & -389 & -.275 & -.071 & -.226 & -167 & .081 & .192 & -.037 & .077 & .611 \\
\hline 39 & .300 & $-.1 \overline{1}$ & .391 & .117 & -.218 & .139 & .156 & -.059 &. .131 & .205 & .439 \\
\hline 40 & .712 & -286 & -.062 & -.066 & .110 & .031 & .151 & -.053 & -1183 & -169 & .685 \\
\hline 41 & .373 & .384 & .116 & -.272 & .057 & .146 & -.180 & -221 & .096 & -160 & .515 \\
\hline 42 & .534 & .380 & -.102 & .245 & .259 & .089 & .150 & .036 & .100 & -.107 & .633 \\
\hline 43 & .510 & .501 & -.212 & .978 & .174 & .099 & .159 & .138 & .151 & -.058 & .744 \\
\hline 44 & .276 & .043 & .307 & -.084 & -.126 & -227 & .062 & .163 & -.114 & -.112 & .303 \\
\hline 45 & .153 & .289 & .286 & -.201 & .052 & -.215 & -.179 & -.029 & .098 & -.140 & .342 \\
\hline 46 & .357 & -.278 & -.089 & .118 & -.131 & -.226 & $-\ldots .238$ & -.068 & .044 & -.190 & .394 \\
\hline 47 & .182 & .392 & -.078 & .337 & .829 & .044 & .060 & .101 & -.111 & -.060 & .446 \\
\hline 49 & .350 & .502 & -180 & -.059 & -305 & .120 & -196 & .082 & -.112 & .164 & .597 \\
\hline 50 & .229 & .255 & -.2930 & .093 & -.224 & -.079 & -.250 & -.110 & -.217 & .206 & .401 \\
\hline 51 & 16 & .421 & -.303 & -.118 & -465 & .180 & .059 & .089 & -.068 & -.056 & .668 \\
\hline 53 & .459 & .283 & .482 & .067 & .069 & -230 & .079 & .087 & .059 & -.069 & .607 \\
\hline 54 & .482 & .296 & .386 & -.090 & .235 & -.192 & .076 & .097 & .135 & .127 & .611 \\
\hline 55 & .485 & -.092 & -.225 & .083 & -.178 & -.047 & -.059 & .190 & $-.12 g$ & .132 & .408 \\
\hline 56 & .535 & -.227 & \pm 47 & .158 & $.06 \overline{\mathrm{j}}$ & -.035 & -.089 & .023 & -.086 & -.149 & .467 \\
\hline
\end{tabular}


JOHN B. CARROLL

TABLE 3

The Final Transformation Matrix $\left(\Lambda_{17}\right)$

\begin{tabular}{rrrrrrrrrrrr}
\hline & $A$ & $B$ & $G$ & $\bar{C}$ & $D$ & $E$ & $F$ & $G$ & $H$ & $J$ & $K$ \\
$I$ & .28 & .15 & .30 & .42 & .32 & .27 & .24 & .34 & .16 & .21 & .04 \\
$I I$ & .01 & -.18 &.- .56 & -.48 & .28 & .09 & .30 & .30 & .22 & -.19 & -.15 \\
$I I I$ & .38 & .37 & -.40 & -.40 & -.21 & -.04 & -.27 & -.18 & .39 & .09 & -.12 \\
$I V$ & .06 & .47 & -.15 & -.22 & .53 & -.14 & .08 & -.39 & -.43 & -.13 & -.10 \\
$V$ & -.29 & -.25 & -.12 & -.07 & .55 & .20 & -.63 & -.20 & .15 & .13 & .17 \\
$V I$ & -.47 & .48 & -.55 & -.50 & -.19 & -.03 & -.14 & .56 & -.94 & .22 & .26 \\
$V I I$ & .54 & -.35 & -.15 & -.13 & .30 & -.57 & -.29 & .26 & -.30 & .07 & .27 \\
$V I I I$ & -.37 & .10 & .10 & .19 & .28 & -.66 & .01 & -.07 & .51 & .19 & .11 \\
$I X$ & .01 & .39 & .49 & .17 & .10 & -.23 & -.41 & .34 & .29 & -.84 & .00 \\
$X$ & .18 & .11 & -.09 & -.22 & .00 & .23 & .31 & -.25 & .11 & -.28 & .88 \\
\hline
\end{tabular}

TABLE 4 The Rotated Factorial

Matrix

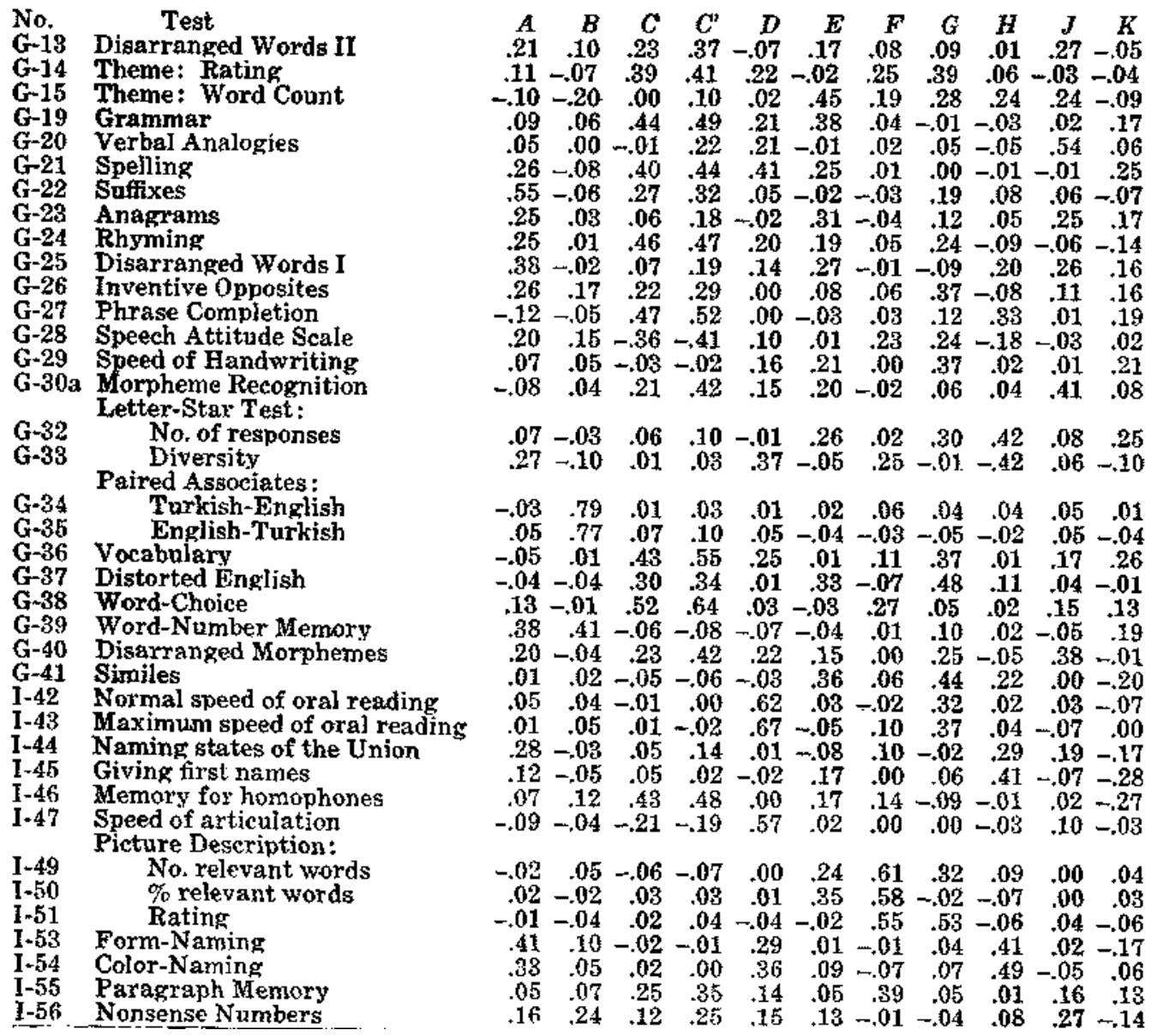


TABLE 5

Cosines of Angles between the Reference Vectors

\begin{tabular}{|c|c|c|c|c|c|c|c|c|c|c|c|}
\hline & $A$ & $B$ & $C$ & $C$ & $D$ & $E$ & 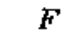 & $G$ & $\boldsymbol{H}$ & $J$ & $\boldsymbol{K}$ \\
\hline$A$ & 1.00 & -.14 & .08 & .02 & .05 & -.02 & .11 & -.07 & -.04 & -.15 & .05 \\
\hline$B$ & -.14 & 1.00 & -.10 & -.22 & -.10 & -.05 & -.01 & .07 & .01 & -.26 & .03 \\
\hline$G$ & .08 & -10 & 1.00 & $\ldots$ & .07 & -.04 & .08 & -.01 & $.2 \%$ & -.40 & -.14 \\
\hline$C$ & .02 & -.22 & & 1.00 & .05 & -.02 & .06 & -.04 & .22 & .00 & -.19 \\
\hline$D$ & .05 & -.10 & .07 & .05 & 1.00 & -.18 & -18 & -.10 & .01 & -.06 & .09 \\
\hline$\vec{E}$ & -.02 & -.05 & -.04 & -.02 & -.18 & 1.00 & .20 & -.12 & -.06 & .03 & .01 \\
\hline$F$ & .11 & -.01 & .03 & .06 & -18 & .29 & 1.00 & -.06 & -.08 & .08 & .04 \\
\hline$G$ & $-.0 \%$ & .07 & -.01 & -.04 & -.10 & -.12 & -.06 & 1.00 & -.04 & -.07 & -.02 \\
\hline $\boldsymbol{H}$ & -.04 & .01 & .27 & 22 & .01 & -.00 & -.08 & -.04 & 1.00 & -17 & -00 \\
\hline$J$ & -.15 & -20 & -.40 & .00 & -.06 & .03 & .08 & -.07 &.$- \mathrm{I} 7$ & 1.00 & -.09 \\
\hline $\boldsymbol{K}$ & .05 & .03 & -.14 & -19 & .09 & .01 & .04 & -.02 & -.02 & -.09 & 1.00 \\
\hline
\end{tabular}

TABLE 6

Correlations between the Primary Factors

a. $R_{1}$, when reference vector $C$ is oblique to $J$.

\begin{tabular}{|c|c|c|c|c|c|c|c|c|c|c|}
\hline & $A$ & $B$ & $C$ & $D$ & $E$ & $F$ & $G$ & $H$ & $J$ & $K$ \\
\hline$A$ & 1.000 & .190 & .019 & -.022 & .073 & -.140 & .076 & .066 & .202 & \\
\hline$B$ & & 1.000 & & .104 & .086 & & -.011 & -00 & .357 & .025 \\
\hline & .019 & $22 \pi$ & 1.000 & -.052 & .050 & -.1 & .020 & $-2]$ & .436 & .203 \\
\hline$D$ & -022 & .104 & -.052 & 1.000 & .151 & .148 & .124 & .0 & .044 & -102 \\
\hline$E$ & .073 & .086 & .056 & .151 & 1.000 & -.265 & .131 & .041 & .065 & -.001 \\
\hline$F$ & $-.1 \div 0$ & -.1058 & -121 & .148 & -265 & 1,000 & .029 & .071 & -.130 & -.075 \\
\hline$G$ & .076 & -.011 & .022 & .124 & .131 &, 029 & 1.000 & .063 & .088 & .016 \\
\hline$H$ & .066 & -.003 & -.922 & $.08 \pi$ & .041 & .071 & .063 & 1.000 & .067 & -.016 \\
\hline$J$ & .202 & .957 & .436 & .044 & .065 & -130 & .088 & .067 & 1.000 & .157 \\
\hline & -.034 & .025 & .203 & -.102 & -.001 & -.075 & .016 & -.016 & .157 & 1.000 \\
\hline
\end{tabular}

b. $R_{1}$, when reference vector $C$ is orthogonal to $J$.

\begin{tabular}{lrrrrrrrrrr} 
& $A$ & $D$ & $C$ & $D$ & $E$ & $F$ & $G$ & $H$ & $J$ & \multicolumn{1}{c}{$K$} \\
$A$ & 1.000 & .189 & .018 & -.022 & .018 & -.140 & .076 & .060 & .216 & -.204 \\
$B$ & .189 & 1.000 & .222 & .104 & .084 & -.054 & -.012 & -.001 & .298 & .023 \\
$C^{\prime}$ & .018 & .222 & 1.000 & -.051 & .040 & -.108 & .018 & -.221 & .048 & .200 \\
$D$ & -.022 & .104 & -.051 & 1.000 & .152 & .148 & .124 & .077 & .071 & -.102 \\
$E$ & .070 & .084 & .040 & .152 & 1.000 & -.263 & .130 & .045 & .048 & -.004 \\
$F$ & -.140 & -.054 & -.108 & .148 & -.263 & 1.000 & .080 & .068 & -.090 & -.072 \\
$G$ & .076 & -.012 & .018 & .124 & .150 & .000 & 1.000 & .065 & .087 & .016 \\
$H$ & .066 & -.001 & -.221 & .027 & .045 & .068 & .068 & 1.000 & .171 & -.016 \\
$J$ & .216 & .208 & .048 & .071 & .048 & -.090 & .087 & .171 & 1.000 & .086 \\
$K$ & -.024 & .023 & .200 & -.102 & -.004 & -.072 & .016 & -.016 & .086 & 1.000
\end{tabular}


TABLE 7 Saturations $\left(a_{k 1}\right)$ of Primaries in a

Second-Order General Factor*

a. From $R_{1}$, with $C$ oblique to $J$.

$\begin{array}{lllllllllllll}A & B & C & D & E & F & G & H & J & K & \\ .234 & .489 & .276 & .131 & .266 & .000 & .190 & .000 & .668 & .000\end{array}$

b. From $R_{p}$ with $C^{\prime}$ orthogonal to $J$.

$\begin{array}{cccccccccc}A & B & C & D & E & F & G & H & J & K \\ .276 & .580 & .100 & .167 & .283 & .000 & .212 & .000 & .437 & .000\end{array}$

$*$ The saturations of primaries $F . H$, and A" have been arbitrarily set equal to zero
because of the many appreciable negative correlations in the corresponding arrays of
Table 6 .

\section{REFERENCES}

Anderson, V. A. The auditory memory span for speech sounds. Speech Monogr., 1938, 5, 115-129.

Carroll, J. B. Diversity of vocabulary and the harmonic series law of word-frequency distribution. Psychol. Rec, 1938, 2, 379-386.

Carroll, J. B. Knowledge of English roots and affixes as related to vocabulary and Latin study. J. educ. Res., 1940, 34, 102-111.

Coombs, C. H. A criterion for significant common factor variance. Psycho-metrika, 1941, 6, 267-272.

Davis, F. C. The functional significance of imagery differences. J. exp. Psychol., 1932, 15, 630-661.

Knower, F. H. A study of speech attitudes and adjustments. Speech Monogr., 1938, 5, 130-203.

Stinchfield, S. M. The formulation and standardization of a series of graded speech tests. Psychol. Monogr., 1923, 33, No. 2.

Stumberg, D. A study of poetic talent. J. exp. Psycho!., 1928, 11, 219-234. Terman, L. M. and Merrill, M. A. Measuring intelligence: A guide to the administration of the new revised Stanford-Binet tests of intelligence. Boston: Houghton Mifflin, 1937.

Thurstone, L. L. The vectors of mind. Chicago: Univ. Chicago Press, 1935. Thurstone, L. L. Primary mental abilities. Psychometric Monogr., 1938, No. 1.

Thurstone, L. L. The perceptual factor. Psychometrika, 1938, 3, 1-17. Thurstone, L. L. A new rotational method in factor analysis. Psychometrika, 1938, 3. 199-218.

Thurstone, L. L. Experimental study of simple structure. Psychometrika, 1940, 5, 153168.

Thurstone, L. L, \& Thurstone, Thelma Gwinn. Factorial studies of intelligence. Psychometric Monogr., 1941, No. 2.

Tucker. L. R. The role of correlated factors in factor analysis. Psychometrika, 1940, 5, 141-152.

Woodworth, R. S., \& Wells, F. L. Association tests. Psychol. Monogr., 1911, 13, No. 57. 